\title{
Speed enhancement in VCSELs employing grating mirrors
}

\author{
Chung, II-Sug; Mørk, Jesper
}

Published in:

Proceedings of SPIE - The International Society for Optical Engineering

Link to article, DOI:

$10.1117 / 12.2005362$

Publication date:

2013

Link back to DTU Orbit

Citation (APA):

Chung, I-S., \& Mørk, J. (2013). Speed enhancement in VCSELs employing grating mirrors. Proceedings of SPIE - The International Society for Optical Engineering, 8633, 863308. https://doi.org/10.1117/12.2005362

\section{General rights}

Copyright and moral rights for the publications made accessible in the public portal are retained by the authors and/or other copyright owners and it is a condition of accessing publications that users recognise and abide by the legal requirements associated with these rights.

- Users may download and print one copy of any publication from the public portal for the purpose of private study or research.

- You may not further distribute the material or use it for any profit-making activity or commercial gain

- You may freely distribute the URL identifying the publication in the public portal

If you believe that this document breaches copyright please contact us providing details, and we will remove access to the work immediately and investigate your claim 


\title{
Speed enhancement in VCSELs employing grating mirrors
}

\author{
Il-Sug Chung* and Jesper Mørk \\ Department of Photonics Engineering, Technical University of Denmark, DK-2800 Kgs. Lyngby, \\ Denmark \\ *ilch@fotonik.dtu.dk
}

\begin{abstract}
In recent years, various approaches to improve the speed of directly modulated vertical-cavity surface-emitting lasers (VCSELs) have been reported and demonstrated good improvement. In this paper, we propose and numerically investigate a new possibility of using high-index-contrast grating (HCG) as mirror for VCSELs. By changing the grating design, one can control the reflection delay of the grating mirror, enabling the control of cavity photon lifetime. On the other hand, short energy penetration depth of the HCG results in smaller modal volume, compared to DBR VCSELs. An example structure shows that the HCG VCSEL has a 30-\% higher 3-dB bandwidth than the DBR VCSEL.
\end{abstract}

Keywords: High speed, vertical-cavity surface-emitting laser, HCG, subwavelength

\section{INTRODUCTION}

For long time, vertical-cavity surface-emitting lasers (VCSELs) have been promoted as a promising light source for short-range optical fiber links such as local area networks (LANs), storage area networks (SANs), passive optical networks (PONs), active optical cables, and recently optical interconnects inside computers. Their many attractive characteristics make them attractive component, including relatively low cost, low energy consumption, high direct modulation speed, circularly symmetric emission profile, broadband wavelength tenability, wavelength scalability $(850$ $\mathrm{nm}, 980 \mathrm{~nm}, 1050 \mathrm{~nm}, 1310 \mathrm{~nm}, 1550 \mathrm{~nm}$ ), and feasible two-dimensional (2D) array.

One of technological advances in recent years is to push the direct modulation speed of VCSELs to beyond $20 \mathrm{Gbps}{ }^{[1]}$ to meet the expected demand on faster lasers for the above mentioned short-distance-link applications. For improvement of extrinsic response speed, parasitic components have been squeezed to very small values by optimizing device structures, e.g., by using tapered oxide layers and thick oxides to reduce the junction capacitance. ${ }^{[2]}$ For improving the intrinsic response speed, several obvious choices have been derived from the relaxation oscillation frequency, $\omega_{R}:^{[3]}$

$$
\omega_{R} \approx \sqrt{\frac{v_{g} a}{q V_{p}} \eta_{i}\left(I-I_{t h}\right)}
$$

One may increase the differential gain, $a$, by optimizing active materials, reduce the modal volume $V_{p}$, increase the injection efficiency, $\eta_{i}$ by optimizing the current injection part, decrease the threshold current $I_{t h}$, and improve the reliability of VCSELs at higher injection levels of current, $I{ }^{[4]}$ Lots of efforts have put into improve all these aspects. Another interesting approach recently reported is to make the photon life time, $\tau_{p}$ shorter. The relation in Eq. (1) can be reformed to

$$
\omega_{R} \approx \sqrt{\frac{v_{g} a N_{p}}{\tau_{p}}}
$$

where $N_{p}$ is photon density. As we will see detail later, the photon life time is related to mirror and internal losses, and roundtrip travel time in the cavity. Thus, by making the cavity length shorter ${ }^{[5]}$ or reducing the reflectivity of an emitting mirror, one may reduce the photon life time, increasing $\omega_{R}$.

In this paper, we propose a new way of improving the speed by employing high-index-contrast subwavelength gratings (HCGs) as mirrors; the HCGs as mirrors for VCSELs can be used to control the photon lifetime and reduce the modal volume of the VCSEL cavity. The proposed way is less overlapped with aforementioned approaches. For example, the 
photon life time can be reduced without decreasing the reflectivity of a mirror, which may increase the threshold current. Thus, the proposed approach may provide additional way of improving intrinsic speed response. Investigations are performed using numerical methods. In Section 2, we derive an expression for cavity photon lifetime, carefully considering the phase penetration length of mirrors, and compare the cavity photon lifetime of a normal VCSEL with double DBRs, with that of a VCSEL with double HCGs. In Section 3, we evaluate the modal volumes of the two VCSELs. In Section 4, we compare and discuss the frequency response functions of the two VCSELs by solving rate equations, finding the photon densities at fixed current, and substituting those results to the small signal model.

\section{CONTROL OF CAVITY PHOTON LIFETIME BY HCGS}

HCGs are a membrane layer with one- or two-dimensional periodic patterns. Typically, the grating period of a HCG should be slightly smaller than the wavelengths to get high reflectivity. The grating is made of a semiconductor or a dielectric material with a high refractive index, and is surrounded by low-refractive-index media. HCGs can exhibit high reflectivity over a broad wavelength range and the high reflectivity can be made polarization-selective. These attractive performance as mirrors make HCGs a strong candidate to replace distribute Bragg reflectors (DBRs) in various photonic and optoelectronic device structures, especially VCSELs. Recently, many novel characteristics of HCG VCSELs have been reported, such as low-threshold,[chang-hasnain] strong single-transverse operation, polarization stability, ultralowenergy-consumption tunability, broadband tunability, fast-tunable laser for optical coherent tomography, and lateral emission capability for silicon photonics light source.

In this section, we will show that HCGs can be used to control the cavity photon lifetime and it will be another interesting property of HCGs. First, an expression for cavity photon life time will be derived, considering the reflection delay time during reflection process in structure mirrors and the penetration length into the structured mirrors.

(a)

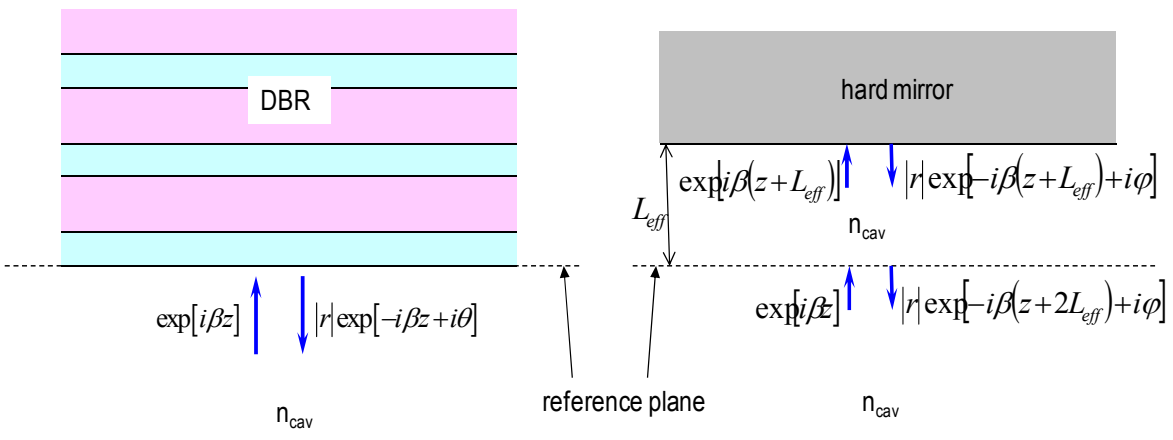

(c)



Figure 1. (a) Waves incident to and reflected from a DBR. (b) A hard mirror model of case (a). (c) Waves incident to and reflected from a HCG. (d) A hard mirror model of case (c) 


\subsection{Photon lifetime in a cavity with structure mirrors}

In a structured mirror like DBRs or HCGs, the reflection process involves propagations within the finite-sized mirror structure, and several internal reflections in the interfaces within the mirror structure. If the cavity length, $L$, i.e., the distance between two mirrors is less than several times of the wavelength of interest, the time taken for the reflection process, hereafter called reflection delay time, should be counted. To consider the role of this reflection delay time in the cavity photon lifetime, the hard mirror model is helpful. Figures 1(a) and 1(b) illustrate the hard mirror model for a DBR. In Fig. 1(a), the reflection phase, $\theta(\lambda)$ includes all information about complex reflection process in the DBR. As shown in Fig. 1(b), in the hard model of the DBR, $\theta(\lambda)$ is modeled as a sum of a round-trip propagation phase by a distance, $L_{\text {eff }}$ and a constant phase $\varphi$. The $L_{\text {eff }}$ is called a phase penetration length. Since $\theta(\lambda)$ linearly varies in the middle of DBR stopband, $L_{\text {eff }}$ can be expressed as, ${ }^{[3]}$

$$
L_{\text {eff }}=-\frac{1}{2}\left[\frac{\partial \theta}{\partial \beta}\right]_{\lambda=\lambda 0}=\frac{1}{4 \pi n_{\text {cav }}}\left[\frac{\partial \theta}{\partial \lambda}\right]_{\lambda=\lambda 0} \lambda_{0}^{2}
$$

where $n_{\text {cav }}$ is the refractive index of the cavity. In the same way, the reflection process in a HCG can be modeled as a hard mirror model, ${ }^{[6],[7]}$ as illustrated in Figs. 1(c) and (d). The reflection delay time, $\tau_{\text {eff }}$ can be estimated by dividing $L_{e f f}$ by the velocity of light in the cavity, here, group velocity, $v_{g}$ :

$$
\tau_{\text {eff }}=\frac{2 L_{e f f}}{v_{g}}
$$

Now, let's consider the photon lifetime, $\tau_{c}$ in a cavity. After $m$-times roundtrip in a cavity, the fraction of number of photons remained in the cavity, compared to the initial number is given by,

$$
\exp \left[-\frac{m \tau_{e f f}^{t o t}}{\tau_{c}}\right]=\left[R_{1} R_{2}\left(1-T_{i}\right)\right]^{m}
$$

where $\tau_{\text {eff }}^{\text {tot }}$ is a total roundtrip time and can be decomposed into,

$$
\tau_{e f f}^{t o t}=\frac{2 L}{v_{g}}+\frac{2 L_{e f f}^{1}}{v_{g}}+\frac{2 L_{e f f}^{2}}{v_{g}}=\frac{2 L_{e f f}^{t o t}}{v_{g}}
$$

where $L, L_{e f f}^{1}, L_{e f f}^{2}$, and $L_{e f f}^{t o t}$ are the nominal cavity length, i.e., the distance between two mirrors, the penetration length of Mirror 1, the penetration length of Mirror 2, and the total effective cavity length, respectively. In Eq. (5), R1 and R2 are the reflectivity of Mirror 1 and Mirror 2, respectively. $T_{i}$ is the fractional loss per pass, i.e., half a roundtrip, due to any types of loss, e.g., diffraction, absorption, etc, and can be expressed in terms of absorption coefficient, $\alpha_{i}$ :

$$
T_{i}=1-\exp \left[-\alpha_{i} L\right]=1-\exp \left[-\left\langle\alpha_{i}\right\rangle\left(L_{\text {eff }}^{1}+L+L_{\text {eff }}^{2}\right)\right]=1-\exp \left[-\left\langle\alpha_{i}\right\rangle L_{\text {eff }}^{\text {tot }}\right]
$$

Here, since the losses in mirrors 1 and 2 are already counted in R1 and R2, we do not need to include any loss during propagation within penetration lengths, absorption coefficients in $L_{\text {eff }}^{1}$ and $L_{\text {eff }}^{2}$. However, for later convenience, we just define $\left\langle\alpha_{i}\right\rangle$ which is often used as an empirical fitting parameter in laser simulations.

After plugging Eq. (6) and (7) into Eq. (5), taking log of the equation gives,

$$
\frac{v_{g}}{\tau_{c}}=-\frac{1}{2 L_{e f f}^{t o t}} \ln \left[R_{1} R_{2}\right]+\left\langle\alpha_{i}\right\rangle
$$


2.2 Cavity photon lifetime for a VCSEL with double DBRs and a VCSEL with double HCGs

(a)

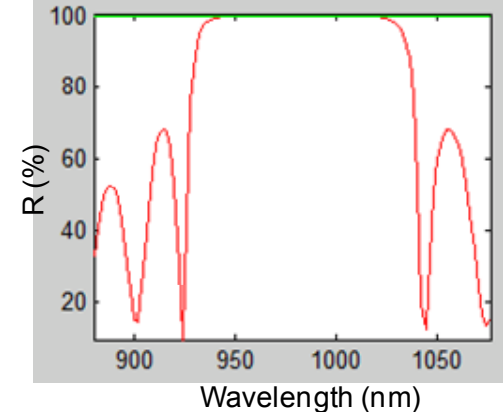

(c)

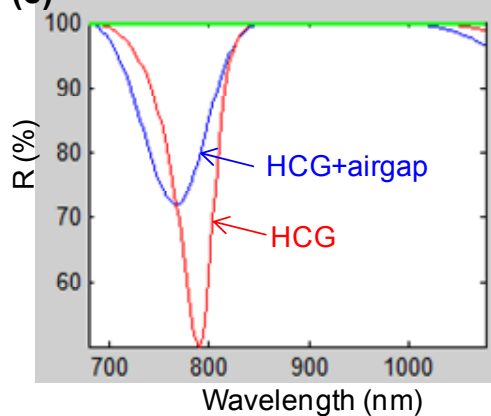

(b)

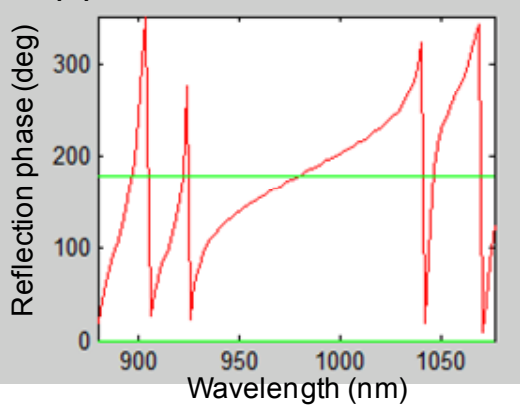

(d)

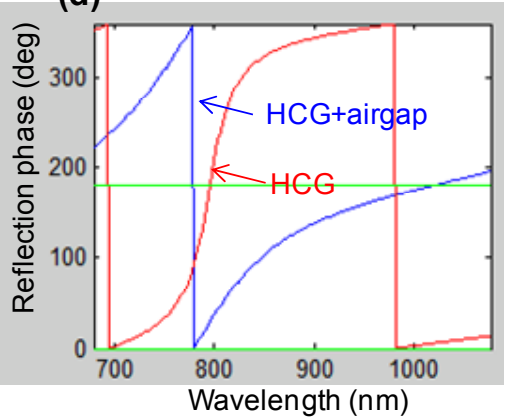

Figure 2. (a) Reflectivity spectrum of a DBR. (b) Reflection phase of the DBR. (c) Reflectivity spectra of a HCG (red) and a $\mathrm{HCG}+$ airgap (blue). (d) Reflection phase spectra of a HCG (red) and HCG + air gap (blue).

(a)

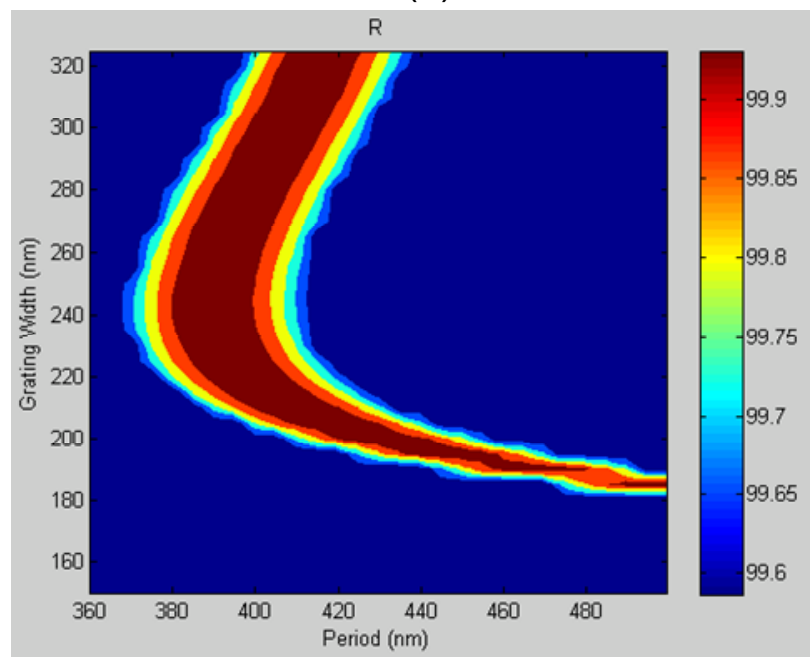

(b)

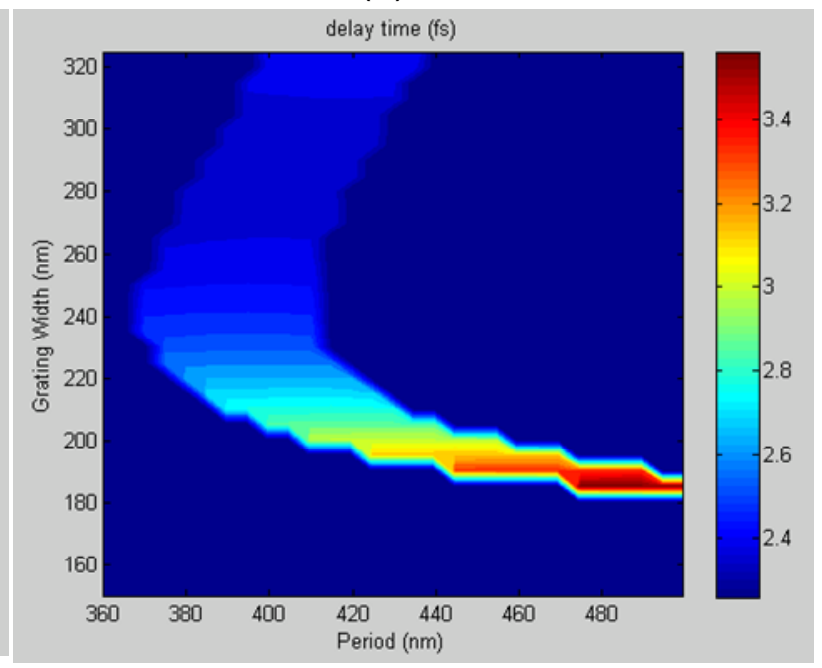

Figure 3. (a) Parametric scanning of reflectivity as a function of grating width and period. (b) Parametric scanning of delay time as a function of grating width and period. 
For comparisons, a VCSEL with double DBRs and a VCSEL with double HCGs are tested. The rigorous coupled wave analysis (RCWA) method is used to calculate the reflectivity and reflection phase of mirrors.

The VCSEL with double DBRs is based on the 980 -nm VCSEL model in [3]. $\left(\mathrm{GaAs} / \mathrm{Al}_{0.9} \mathrm{Ga}_{0.1} \mathrm{As}\right)^{\mathrm{N}} \mathrm{DBR}$ is assumed. As shown in Fig. 2(a), the reflectivity is high enough, and from Fig. 2(b), the penetration depth is calculated to be $485.8 \mathrm{~nm}$. If the number of pairs, $\mathrm{N}$ is more than 25 , the penetration depth is almost same.

To understand the dependence of penetration length (or delay time), parametric scanning of reflectivity and delay time are performed as a function of grating width and grating period. The results are presented in Figs. 3(a) and 3(b). These show that the delay time can be chosen while keeping the reflectivity high. Thus, there is a chance of engineering the cavity photon lifetime without loss of reflectivity. We have chosen a grating period of $400 \mathrm{~nm}$, a grating width of $290 \mathrm{~nm}$, and a grating layer thickness of $241 \mathrm{~nm}$; this combination gives the smallest penetration length of $201.5 \mathrm{~nm}$

The penetration depths of DBR and HCG with an air gap are $485.8 \mathrm{~nm}$ and $201.5 \mathrm{~nm}$, respectively.

\section{CONTROL OF MODAL VOLUME BY HCGS}

In structured mirrors, there are two penetration lengths defined; the phase penetration length as discussed in Section 2 and the power penetration length. The former is related to phase, delay time, longitudinal mode spacing, and free spectral range. The latter is related to modal volume, quantum confinement factor. In DBR case, both lengths might be similar. However, in HCG case, they can be considerably different, in general.

In this paper, first we estimate the quantum confinement factor from field distribution and then calculate the modal volume, $V_{p}$ by dividing active volume $V$ with the quantum confinement factor, $\Gamma$. For calculation of the confinement factor, following relation can be used.

$$
\Gamma=\frac{\int\left|E^{+}+E^{-}\right|^{2} d V}{\int\left|E_{T}^{+}\right|^{2}+\left|E_{T}^{+}\right|^{2} d V}
$$

The confinement factors for the DBR VCSEL and the HCG VCSEL are 0.038 and 0.067, respectively. Accordingly, the volume volume for the DBR and the HCG VCSELs are $6.28 \times 10^{-11} \mathrm{~cm}^{-3}$ and $3.58 \times 10^{-11} \mathrm{~cm}^{-3}$, respectively.

\section{FREQUENCY RESPONSE}

From Sections 2 and 3, we have obtained the photon life time, the modal volume, and the confinement factor for the DBR and the HCG VCSELs. For getting the small-signal frequency response function, we need to photon density, $\mathrm{N}_{\mathrm{p}}$ at a specific injection current. Using laser parameters provided in Ref. [3] and parameters obtained from Sections 2 and 3, we solved the rate equations and obtained the static characteristics, as presented in Fig. 4. The HCG VCSEL has slightly lower threshold current and larger differential efficiency. Thus, the HCG VCSEL has higher photon densities at all bias points. At $\mathrm{I}=2 \mathrm{~mA}$, the DBR and the HCG VCSELs have $\mathrm{N}_{\mathrm{p}}=2.28 \times 10^{14} \mathrm{~cm}^{-3} 3$ and $2.50 \times 10^{14} \mathrm{~cm}^{-3}$, respectively. 

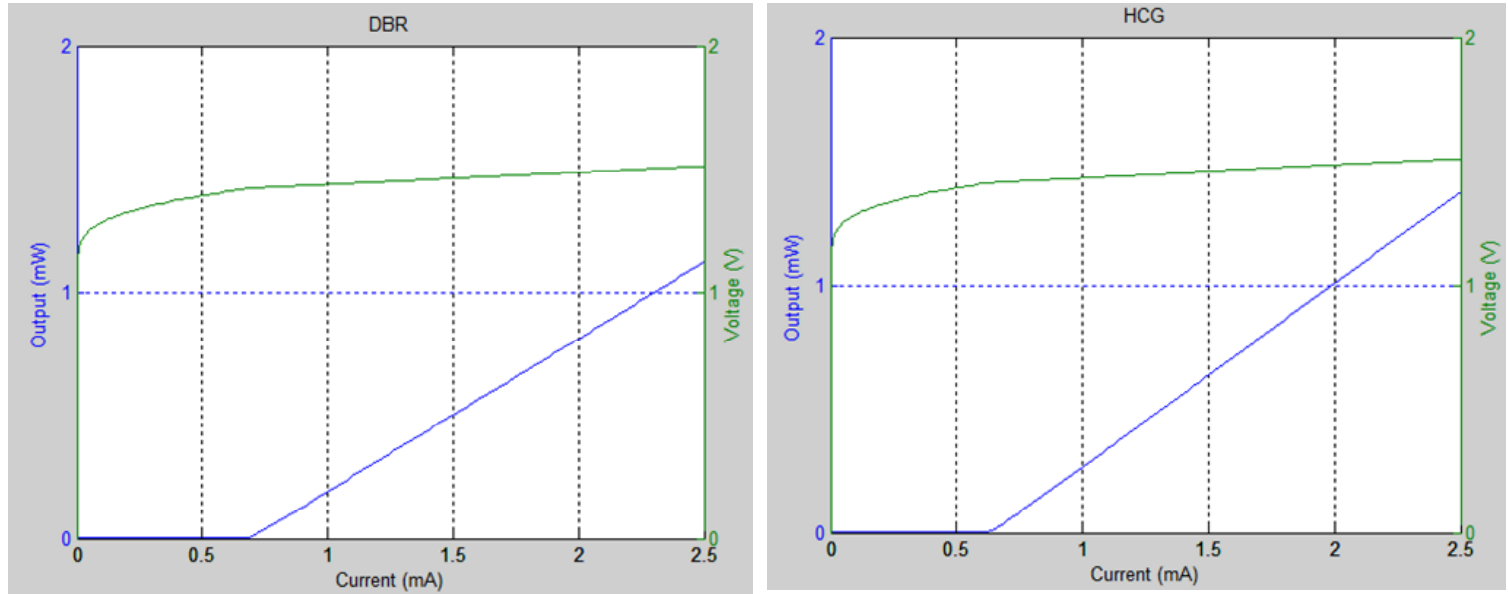

Figure 4. (a) Output power (green line) and voltage (blue line) versus injection current for the VCSEL with double DBRs. (b) Same graphs for the VCSEL with double HCGs.

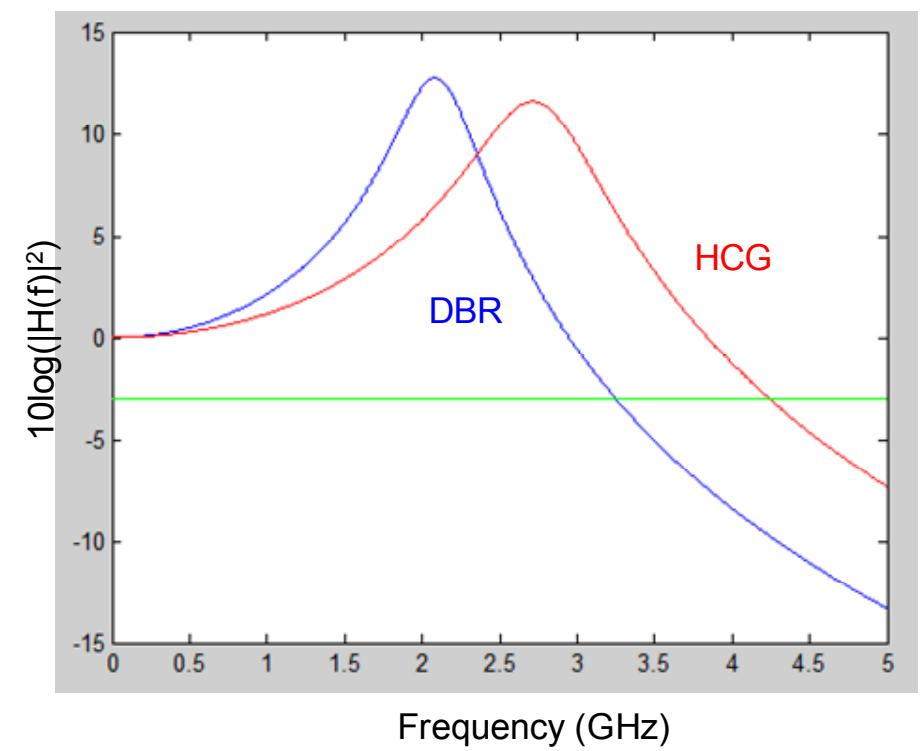

Figure 5. Small signal frequency response of the VCSEL with DBRs (red line) and that of the VCSEL with HCGs (blue line).

Then, the relaxation oscillation frequency, $f_{R}$ and damping factor, $\gamma$ can be evaluated. The DBR and the HCG VCSELs have the relaxation oscillation frequency of $2.11 \mathrm{GHz}$ and $2.76 \mathrm{GHz}$, respectively. The damping factors for the DBR and the HCG VCSELs are $0.49 \mathrm{GHz}$ and $0.73 \mathrm{GHz}$, respectively. As expected from these values, the HCG VCSEL has a larger 3-dB frequency and a smaller oscillation peak.

\section{CONCLUSION}

We have shown that by using HCG the photon lifetime and the modal volume are engineered. Physically, the photon lifetime control relies on the change of reflection delay time. The photon lifetime and the modal volume are related to phase and the energy penetration depths, respectively. Since the control of photon life time is less overlapped with previously reported approaches to increase the intrinsic response speed and can be achieved without loss of reflectivity 
of a mirror, further study of this property may contribute to further improve the direct modulation speed of VCSELs employing the $\mathrm{HCG}$ as a mirror.

\section{REFERENCES}

[1] Hofmann, W. et al., "22-Gb/s long wavelength VCSELs,” Optics Exp., 17, 17547 (2009).

[2] Chang, Y.-C., and Coldren, L. A., "Efficient, high-date-rate, tapered oxide-aperture vertical-cavity surfaceemitting lasers," IEEE J. Sel. Top. Quant. Electron. 15, 704 (2009).

[3] Coldren, L. A., Corzine, S. W., and Mašanović, [Diode lasers and photonic integrated circuits, $2^{\text {nd }}$ edition], Wiley (2012).

[4] Ji, C., Wang, J., Söderström, D. and Giovane, L., "20-Gb/s 850-nm oxide VCSEL operating at $25{ }^{\circ} \mathrm{C}-70{ }^{\circ} \mathrm{C}$," IEEE Photon. Technol. Lett. 22, 670 (2010).

[5] Müller, M., Hofmann, W., Böhm, G., and Amann, M.-C., "Short-cavity long-wavelength VCSELs with modulation bandwidth in excess of 15 GHz," IEEE Photon. Technol. Lett. 21, 1615 (2009).

[6] Chung, I.-S., Iakovlev, V., Sirbu, A., Mereuta, A., Caliman, A., Kapon, E., and Mørk, J., "Broadband MEMStunable high-index-contrast subwavelength grating long-wavelength VCSEL," IEEE J. Quant. Electron. 46, 1245-1253 (2010).

[7] Zhao, D., Ma, Z., and Zhou, W., "Field penetrations in photonic crystal fano reflectors," Optics Exp. 18, 14152 (2010).

[8] Svelto, O., [Principles of lasers, $5^{\text {th }}$ edition], Springer, 170 (2010) 